\title{
Distribution and host plants of Drosophilidae (Diptera) species detected in fruit orchards of the Eastern Mediterranean Region of Turkey
}

Türkiye'nin Doğu Akdeniz Bölgesi'nde meyve bahçelerinde tespit edilen Drosophilidae (Diptera) türlerinin dağılımı ve konukçuları

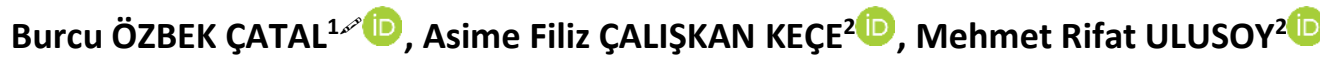

${ }^{1}$ Çukurova University, Pozantı Vocational School, Department of Plant and Animal Production, Pozantı, Adana.

${ }^{2}$ Çukurova University, Department of Plant Protection, Faculty of Agriculture, Balcall, Adana.

MAKALE BILGISI / ARTICLE INFO

Makale tarihçesi / Article history:

DOI: $10.37908 /$ mkutbd.873838

Geliş tarihi /Received:03.02.2021

Kabul tarihi/Accepted:24.06.2021

\section{Keywords:}

Drosophilidae, Drosophila, Zaprionus, Turkey.

Corresponding author: B. ÖZBEK ÇATAL

$\triangle$ : bozbek@cu.edu.tr

\section{ÖZET / A B STR A C T}

\section{Atıf / Citation: Özbek Çatal B, Çalişkan Keçe AF, Ulusoy MR (2021) Distribution and host plants of Drosophilidae (Diptera) species detected in fruit orchards of the Eastern Mediterranean Region of Turkey. MKU. Tar. Bil. Derg. 26(2) : 431-442. DOI: $10.37908 /$ mkutbd. 873838}

\section{INTRODUCTION}

The Drosophilidae is a large cosmopolitan family of acalyptrate flies. Most of the species are saprophagous and feed on microorganisms in spoiled fruits, slime fluxes, fungi or other decaying organic matter. Some occur in flowing tree sap or in flowers. There are more than 4500 Drosophilidae species in the world (Bächli, 2020). The Palaearctic Region is an important area for studying the geographic distribution of drosophilid flies (Bächli and Rocha Pité, 1984). The Palaearctic fauna currently comprises 482 species of 27 genera (Brake and Bächli, 2008). The Drosophilid fauna of the Middle East, excluding Turkey, Israel and Lebanon (Máca, 1987; Bächli et al., 2002), is poorly known. The genus Drosophila contains nearly half of the 4,000 species in the family Drosophilidae. This genus has adapted and dispersed in various niches (Markow and O'Grady, 2008), and presently separated into nine subgenera ( $\mathrm{O}^{\prime} \mathrm{Grady}$ and DeSalle, 2018). The largest subgenus is Drosophila Fállen.

Turkey with its varied climatic and diverse physiographic conditions ensure large number of natural environment for colonisation by Drosophilidae. In Turkey, 36 species belonging to six genera of Drosophilidae have been reported (Koçak and Kemal, 2013). They were not considered to be a threat for the agriculture in Turkey until recent years. However, after detecting the invasive species such as Drosophila suzukii (Matsumura) and Zaprionus indianus (Gupta), which caused significant 
economic losses in fruit production in our country, the interest in this family has increased (Orhan et al., 2016; Catal et al., 2019).

Purpose of the study was to check the existence of the Drosophilidae in fruit orchards of the Eastern Mediterranean Region, Turkey.

\section{MATERIALS and METHODS}

Surveys were carried out various fruit orchards in the Eastern Mediterranean Region (Adana, Hatay, Kahramanmaraş, Mersin, Osmaniye) of Turkey between 2016 and 2019 (Figure 1). The presence of a drosophilid species was determined at diverse locations. Traps baited with $50 \%$ apple cider vinegar and $8-10$ holes no larger than $3 \mathrm{~mm}$ were placed to fruit trees and also samples were taken from the apple cider vinegar traps hung by the producers in the orchards. In addition, infected fruits were collected and placed in the plastic boxes to obtain adult flies. All drosophilid specimens were placed in $70 \%$ ethanol for further identification. All samples were studied and deposited in the Nedim Uygun Biological Control Laboratory of Department of Plant Protection, Faculty of Agriculture, Cukurova University, Adana, Turkey. Identification of the species was made by us and confirmed by Dr. Amir YASSIN (CNRS researcher, Institut de Systématique, Evolution, Biodiversité / France).

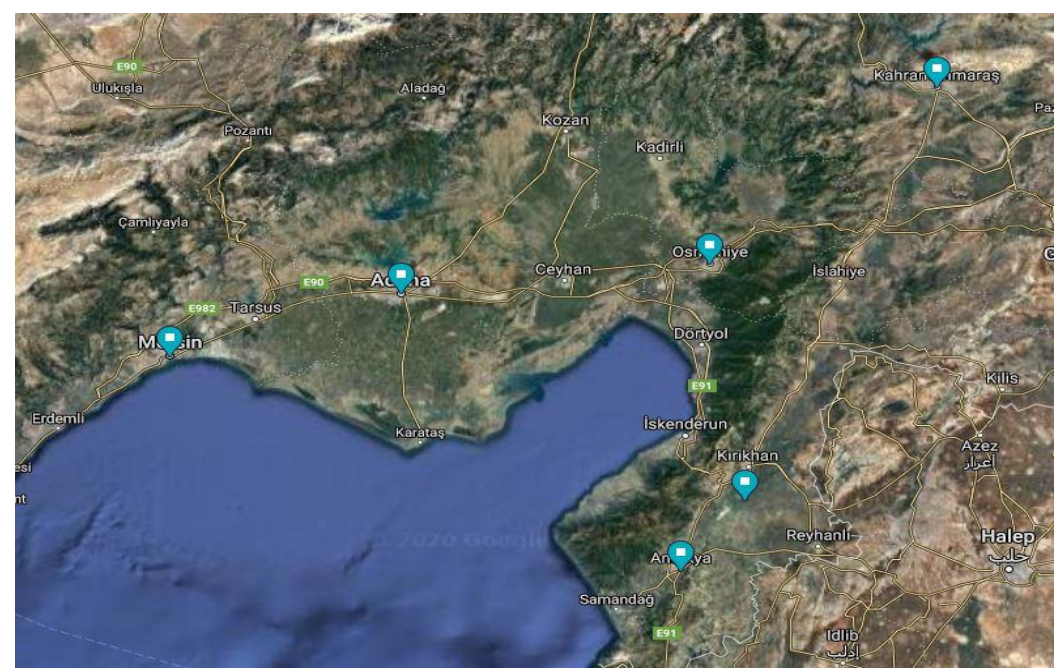

Figure 1. Locations sampled for Drosophilidae species (satellite image from Anonymous, 2020).

\section{RESULTS and DISCUSSION}

Turkey has varied climatic and diverse physiographic conditions and hence is very important for studying a large number of natural environments for colonisation by Drosophilidae. Drosophilids has drawn the attention of researchers for over five decades (Dobzhansky, 1965; Lewontin, 1965; David and Tsacas, 1981; Parsons, 1983), and it is well known that they can be used as models in works of biological invasions (Gibert et al., 2016). As a result of the study, in the material examined list, 11 species in 4 genera were identified. Two genera belong to the subfamily Drosophilinae (Drosophila Fallén, 1823 and Zaprionus Coquillett, 1901) and two belong to Steganinae (Leucophenga Mik, 1886 and Gitona Meigen, 1830). In the results, Drosophila immigrans (Sturtevant), D. melanogaster (Meig.), D. subobscura (Collin), D. suzukii (Matsumura), Zaprionus indianus (Gupta) and $Z$. tuberculatus (Gupta) were found to be common. Virtually all species recorded here are extensively dispersed not only in Turkey but also in other regions of the world (Bächli, 2020).

The distribution and hosts of all the identified species are given in the examined material (Figure 2). The species were listed in alphabetical order.

Family: Drosophilidae

Subfamily: Drosophilinae

Genus: Drosophila Fallén, 1823

\section{Drosophila busckii Coquillett, 1901}

Material examined: Adana, Pozantı, Alpu, Prunus avium L. (Rosaceae), 04.V.2018, 19, 10'; Pozantı, Belemedik, P. avium, 17.V.2019, 2\%क; Pozantı, Hamidiye, P. avium, 13.VI.2018, 2\%o, 10'; Pozantı, Karakışlakçı, Fragaria $x$ ananassa Duch. (Rosaceae), 09.V.2019, 19; Pozantı, Karakışlakçı, P. armeniaca L., 09.V.2019, 10'; Pozantı, Belemedik, P. persica (L.) Batsch, 28.XII.2019, 10'; Mersin, P. domestica L., 28.VI.2018, 29\%, 10'; Mersin, $P$. persica, 28.VI.2018, 10'; Mersin, Vitis vinifera L. (Vitaceae), 13.VII.2018, 19; Çayıryurt, P. persica, 13.VII.2018, 19, 10; Kızılbağ, P. persica, 13.VII.2018, 1 ㅇ․ 
Host: Aphelandra micans Moritz ex Vatke (Acanthaceae), Malus domestica Borkh. (Rosaceae), Musa sp., P. armeniaca, P. avium, Pyrus communis L. (Rosaceae), V. vinifera (Watabe et al., 1993; Zengin and Karaca, 2019; Zengin, 2020).

Distribution: Caucasus, Cosmopolitan, China, European part, Far East., Russia: Siberia (Toda, 1986; Watabe et al. 1993; Ivannikov and Zakharov, 1994; Ivannikov et al., 1998).

Distribution in Turkey; Mersin, Şanlıurfa, Uşak (Koçak and Kemal, 2013; Zengin and Karaca, 2019).

\section{Drosophila hydei Sturtevant, 1921}

Material examined: Adana, Yüreğir, Kadıköy mah., Citrus sinensis L. (Rutaceae), 30.V.2017, 2\%o, 10'; Yüreğir, Kadıköy mah., C. reticulata L., 30.V.2017, 19, $30^{\prime \prime} 0^{\text {; }}$ Yüreğir, Kadıköy mah., C. paradisi L., 30.V.2017, 19; Pozantı, Alpu, P. avium, 01.VI.2017, 19, 10'; Karşıyaka, P. persica, 30.VI.2017, 20'0'; Merkez, Fragaria x ananassa, 24.IV.2018, 1\%; Pozantı, Belemedik, P. avium, 17.V.2019, 2\%ᄋ, 10'; Pozantı, Karakışlakçı, P. avium, 05.XII.2019, $20^{\top} 0^{\pi}$; Pozantı, Belemedik, P. persica, 28.XII.2019, 2\%o; Mersin, P. persica var. nucipersica (L.) C.K. Schneid., 15.VI.2018, 20"0'; Mersin, P. domestica, 21.VI.2018, 2\%ᄋ, 10'; Mersin, P. persica, 21.VI.2018, 19, 20'0'; Çayıryurt, $P$. persica, 13.VII.2018, 4\%ᄋ, 20"0'; Huzurkent, P. domestica, 13.VII.2018, 19, 30'0'; Huzurkent, V. vinifera, 13.VII.2018, 3\%क; Mersin, P. cerasus, 16.VII.2018, 2\%क; Mersin, V. vinifera, 16.VII.2018, 10; Mersin, Rubus fruticosus L. (Rosaceae), 16.VII.2018, 2\%क, $10^{\prime}$.

Host: Caltha arctica R.Br. (Ranunculaceae), citrus garden, Ficus carica L. (Moraceae), Musa sp., Puccinellia phryganoides (Trin.) Scribn. \& Merr. (Poaceae) (Watabe et al., 1993; Ebejer, 2001; Gençer et al., 2005; Nartshuk, 2014).

Distribution: Caucasus, Cosmopolitan, European part, Far East., Russia, West Siberia (Ivannikov and Zakharov, 1994; Ivannikov et al., 1998; Nartshuk, 2014).

Distribution in Turkey. Bursa (Gençer et al., 2005).

\section{Drosophila immigrans Sturtevant, 1921}

Material examined: Adana, Balcalı, P. persica var. nucipersica, 10.V.2017, 5\%क, 40' $0^{\pi}$; Pozantı, Alpu, P. avium, 25.V.2017, 3\%ᄋ, 10'; Yüreğir, Kadıköy mah., C. sinensis, 30.V.2017, 4ㅇ, 20"0'; Yüreğir, Kadıköy mah., C. paradisi, 30.V.2017, 4\%ᄋ, 50 $0^{\top}$; Yüreğir, Kadıköy mah., C. reticulata, 30.V.2017, 5\%क, 60"0'; Tufanbeyli, Merkez, P. avium, 17.VII.2017, 4ㅇ, 10; Merkez, Fragaria $x$ ananassa, 24.IV.2018, 1ㅇ, 40 $0^{\top} 0^{\top}$; Balcalı, C. paradisi,
30.IV.2018, 6\%९, 80"0'; Aladağ, P. avium, 07.VI.2018, 3\%ᄋ, 20 $0^{\top} 0^{\top}$; Saimbeyli, R. fruticosus, 24.VII.2018, 19, $20^{\top} 0^{\top}$; Pozantı, Belemedik, P. avium, 28.V.2019, 2कo; Pozantı, Hamidiye, P. avium, 13.VI.2019, 2\%o, 10'; Pozantı, Belemedik, P. persica, 28.XII.2019, 4ㅇ, 20'0'; Kahramanmaraş, Andırın, Çiğsar, P. avium, 19.VıI.2018, 2\%९, 10'; Mersin, P. persica var. nucipersica, 15.VI.2018, $30^{\prime \prime} 0^{\pi}$; Kepirli, P. domestica, 13.VII.2018, 2\%o, 30"0'; Çayıryurt, P. persica, 13.VII.2018, 2\%ᄋ, 20 $0^{\top} \sigma^{\top}$; Çayıryurt, V. vinifera, 13.VII.2018, 300 $0^{n}$; Kızılbağ, $P$. persica, 13.VII.2018, 19, 20 $0^{\mathrm{N}}$; Huzurkent, V. vinifera, 13.VII.2018, 19, 10'; Mersin, P. cerasus, 16.VII.2018, 29o, 10"; Mersin, P. domestica, 16.VII.2018, 49ᄋ, 30" $0^{\prime \prime}$; Mersin, R. fruticosus, 16.VII.2018, 5\%क, $20^{\top} 0^{\top}$.

Host: Citrus garden, F. carica, Musa sp., P. communis, V. vinifera (Watabe et al., 1993; Ebejer, 2001; Gençer et al., 2005; Zengin and Karaca, 2019).

Distribution: Azerbaijan, Caucasus, China, Cosmopolitan, European part, Far East., Georgia, Russia: West Siberia, East Siberia (Sturtevant, 1942; Watabe et al., 1993; Ivannikov and Zakharov, 1994; Ivannikov et al., 1998; Oboňa et al., 2019).

Distribution in Turkey. Bursa, Mersin, Trabzon, Uşak (Gençer et al., 2005; Koçak and Kemal, 2013; Zengin and Karaca, 2019).

Drosophila immigrans, a cosmopolitan species within the subgenus of Drosophila is abundant in the Nearctic and Palearctic Regions (David and Tsacas, 1981) and have been wide in our region. It has been observed that $D$. immigrans continues to be seen in nature at high altitudes with the cooling of the weather in our region. It has also been reported by researchers that the plenty of $D$. immigrans appears low in warmer regions, but increases in colder southern regions (Sene et al., 1980; Hochmüller et al., 2010).

\section{Drosophila melanogaster Meigen, 1830}

Material examined: Adana, Balcalı, Morus alba L.

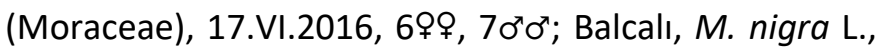
17.VI.2016, 19\%o, 130'o'; Pozantı, Alpu, 20.VII.2016, P. domestica, 9ㅇ, 110 $0^{\top}$; Pozantı, Alpu, M. nigra,

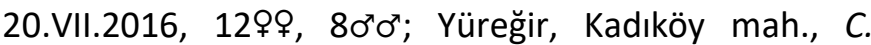
sinensis , 30.V.2017, 4\%ᄋ, 20 $\sigma^{\top}$; Yüreğir, Kadıköy mah., C. paradisi, 30.V.2017, 3ㅇ; Yüreğir, Kadıköy mah., C. reticulata, 30.V.2017, 19, 60" $0^{\pi}$; Pozantı, Alpu, P. avium, 01.VI.2017, 8\%9, 90'0'; Tufanbeyli, Merkez, P. avium, 17.VII.2017, 4\%ᄋ, 20'0'; Merkez, Fragaria $x$ ananassa, 24.IV.2018, 5ㅇ, 160"0'; Balcalı, P. avium, 04.V.2018, 13ㅇ, 170 $10^{\pi}$; Balcalı, Eriobotrya japonica Lindl. 
(Rosaceae), 20.V.2018, 4\%ᄋ, 50 $0^{\top}$; Balcalı, C. paradisi, 20.V.2018, 7\%ᄋ, 60 $0^{\pi}$; Karaisalı, Etekli, P. avium, 01.VI.2018, 2\%o, 10;; Karaisalı, Başpınar, M. alba, 01.VI.2018, 3ㅇ, 30 $30^{\top}$; Aladağ, Değirmencik, P. avium, 07.VI.2018, 3\%९, 2000; Saimbeyli, Merkez, P. avium, 29.VI.2018, 7\%ᄋ, 90'0;; Saimbeyli, Merkez, M. alba, 29.VI.2018, 5\%९, 30"0'; Saimbeyli, Merkez, P. domestica, 29.VI.2018, 4\%ᄋ, 10'; Saimbeyli, Gürleşen, P. avium, 29.VI.2018, 2कo, 10;; Saimbeyli, Obruk, P. avium, 29.VI.2018, 5\%क; Saimbeyli, Merkez, R. fruticosus, 24.VII.2018, 39ᄋ, 4ơo'; Saimbeyli, Kalesekisi, M. alba, 24.VII.2018, 5\%९, 20"0'; Saimbeyli, Kalesekisi, P. avium,

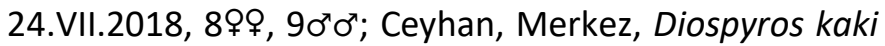
L. (Ebenaceae), 01.VIII.2018, 3\%ᄋ, 10'; Pozantı, Belemedik, P. avium, 17.V.2019, 4\%9, 90'o'; Pozantı, Aşçıbekirli, P. avium, 26.VI.2019, 3\%o; Pozantı, Hamidiye, P. avium, 09.VII.2019, 3\%९, $20^{\top} \sigma^{\top}$; Pozantı, Hamidiye, M. domestica, 09.VII.2019, 8000'; Pozantı, Karakışlakçı, $P$.

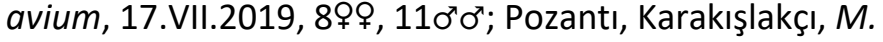
domestica, 17.VII.2019, 5\%ᄋ, 60"0'; Pozantı, Karakışlakçı, Fragaria $x$ ananassa, 17.VII.2019, 119ᄋ, 60" $0^{\prime \prime}$; Pozantı,

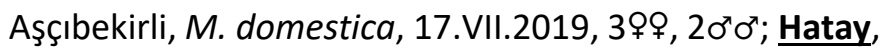
Antakya, Merkez, F. carica, 22.VII.2018, 10\%ᄋ, 120"0; Antakya, P. persica, 22.VII.2018, 5\%क, 90' $0^{\top}$; Defne, F. carica, 22.VII.2018, 5\%, 100 $0^{\top}$; Kahramanmaraș, Geben, P. cerasus, 19.VII.2018, 4\%क, 50 $0^{\top}$; Andırın, Çiğsar, P. avium, 19.VII.2018, 6\%̊, 70'ơ; Andırın, Çokak,

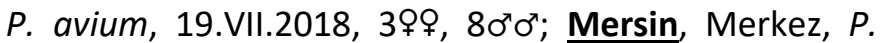
persica var. nucipersica, 15.VI.2018, 29\%, 60" $0^{\top}$; Merkez, P. domestica, 21.VI.2018, 40'0'; Merkez, P. persica, 28.VI.2018, 7\%ᄋ, 90'0'; Merkez, R. fruticosus,

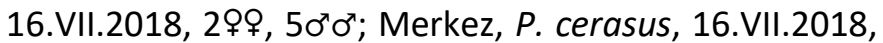

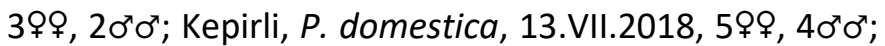
Kepirli, M. nigra, 13.VII.2018, 7\%क, 110 $0^{\pi}$; Değnek, $P$. avium, 13.VII.2018, 5\%9, 30"o'; Değnek, M. nigra, 13.VII.2018, 5\%९, 70'0'; Kızılbağ, P. persica, 13.VII.2018, 4ㅇ, 20 $\sigma^{\prime} \sigma^{\prime}$; Huzurkent, V. vinifera, 13.VII.2018, 19, 10'; Değirmençay, $P$. persica, 13.VII.2018, 3\%ᄋ, 40"0'; Çayıryurt, V. vinifera, 13.VII.2018, 4\%ᄋ; Çayıryurt, $P$. communis, 13.VII.2018, 19, 30"0'; Çayıryurt, P. persica, 13.VII.2018, 19, 40"0'; Gözne, 13.VII.2018, 20'0'; Osmaniye, Merkez, F. carica, 01.VIII.2018, 7\%ᄋ, 90'0'; Merkez, V. vinifera, 01.VIII.2018, 39\%; Merkez, R. fruticosus, 01.VIII.2018, 5\%, 30"0".

Host: Citrus garden, F. carica, Musa sp., Myrica rubra (Lour.) Siebold and Zucc. (Myricaceae), P. avium, P. communis, V. vinifera (Watabe et al., 1993; Ebejer, 2001;
Gençer et al., 2005; Guo, 2007; Li et al., 2011; Zengin and Karaca, 2019).

Distribution: Cosmopolitan, China, Russia: West Siberia, European part, Caucasus, East Siberia, Far East (Sturtevant, 1942; Okada, 1954; Watabe et al., 1993; Ivannikov and Zakharov, 1994; Ivannikov et al., 1998).

Distribution in Turkey. Adana, Bursa, İstanbul, Mersin, Uşak (Gençer et al., 2005; Koçak and Kemal, 2013; Catal et al., 2018; Zengin and Karaca, 2019).

Drosophila melanogaster is one of the dominant species in the survey area. Since the survey area was very close to the residential area and agricultural area in the study carried out by Gleason et al. (2019), this species were found to be the dominant species in the traps. Also, $D$. melanogaster and $D$. suzukii are tropical species. Since the resistance of tropical drosophilid species to the winter cold is weaker than that of the other drosophilid species, these species prefer the sheltered locations, which are close to the residential areas, in order to survive the winter (Hoffmann et al., 2003; Langille et al., 2016). The African species D. melanogaster is one of the most studied drosophilid species (Capy and Gibert, 2004).

\section{Drosophila subobscura Collin, 1936}

Material examined: Adana, Pozantı, Alpu, P. avium, 25.V.2017, 4\%o, 20'o'; Yüreğir, Kadıköy mah., C. reticulata, 30.V.2017, 5\%क, 30 $0^{\top} 0^{\top}$; Tufanbeyli, Merkez, $P$. avium, 17.VII.2017, 19, 200'; Merkez, Fragaria $x$ ananassa, 24.IV.2018, 1\%, 40 $\sigma^{\top} \sigma^{\top}$; Pozantı, Hamidiye, $P$. avium, 13.VI.2018, 2\%9, 10'; Saimbeyli, Merkez, P. avium, 29.VI.2018, 20'0'; Saimbeyli, Obruk, P. avium, 29.VI.2018, 19, 10'; Pozantı, Belemedik, P. avium, 02.I.2019, 5o̊; Pozantı, Aş̧̧ıekirli, M. domestica, 05.XII.2019, 19; Pozantı, Karakışlakçı, M. domestica, 05.XII.2019, 10'; Pozantı, Belemedik, P. persica, 28.XII.2019, 3ㅇ, 20"0'; Kahramanmaraş, Andırın, Çiğsar, P. avium, 19.VII.2018, 3\%क, 10'; Mersin, Kızılbağ, $P$. persica var. nucipersica, 15.VI.2018, 200 $0^{\pi}$; Değnek, $P$. avium, 13.VII.2018, 4ㅇ, 30 $0^{\pi}$; Kızılbağ, P. persica, 13.VII.2018, 39ᄋ, 200' ; ; Huzurkent, P. communis, 13.VII.2018, 19, $10^{2}$.

Host: Citrus garden, $P$. communis, V. vinifera (Ebejer, 2001; Zengin and Karaca, 2019).

Distribution: Africa, Azores, Europe, Chile, China, Madeira (Bächli and Burla, 1985; Watabe et al., 1993).

Distribution in Turkey. Adana, Antalya, Bolu, Mersin, Samsun, Trabzon, Uşak, Zonguldak (Koçak and Kemal, 2013; Catal et al., 2018; Zengin and Karaca, 2019). Drosophila subobscura was observed in the traps since 
from January to December and remained the most frequently detected species. As a result of the studies performed, it was reported that a vast majority of Drosophila species spent the winter as adult, but some others might overwinter in the larvae, pupa, or reproductive diapause (Stephens et al., 2015). In contrast, since $D$. subobscura winters without diapause (Goto et al., 1999), it is thought to be seen in the early seasons compared to other species.

\section{Drosophila suzukii Matsumura, 1931}

Material examined: Adana, Balcalı, M. alba, 17.VI.2016,

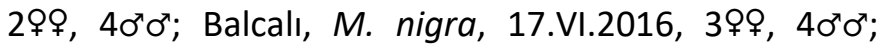
Balcalı, C. paradisi, 17.VI.2016, 5\%क, 90' $0^{\top}$; Balcalı, P. avium, 04.V.2018, 6\%\%, 70'o'; Ceyhan, P. avium, 14.V.2018, 3\%o, 20'0'; Balcalı, E. japonica, 20.V.2018, 40"o'; Pozantı, Alpu, P. avium, 27.V.2018, 2\%ᄋ, 50"0'; Pozantı, Alpu, Fragaria x ananassa, 27.V.2018, 6\%ᄋ, 50'0'; Karaisalı, Merkez, M. alba, 01.VI.2018, 10'; Karaisalı, Başpınar, M. alba, 01.VI.2018, 10'; Aladağ, Merkez, P. avium, 07.VI.2018, 2\%क; Aladağ, Değirmencik, P. avium, 07.Vl.2018, 19, 10'; Pozantı, Hamidiye, P. avium, 13.VI.2018, 4\%ᄋ, 70'ơ; Saimbeyli, Merkez, P. avium, 29.Vl.2018, 2\%o; Saimbeyli, Obruk, P. avium, 29.VI.2018, 3\%o; Saimbeyli, Gürleşen, P. avium, 29.VI.2018, 2\%o, 10; Tufanbeyli, Merkez, M. alba, 29.VI.2018, 2\%\%; Tufanbeyli, Merkez, P. avium, 29.VI.2018, 3\%क; Saimbeyli, Kalesekisi, M. alba, 24.VII.2018, 1ㅇ, 10'; Saimbeyli, Kalesekisi, P. avium, 24.VII.2018, 3\%ᄋ, 10'; Yüreğir, Kadıköy mah., C. sinensis, 07.VII.2018, 4\%ᄋ, 20"0'; Ceyhan, D. kaki, 01.VIII.2018, 2\%९, 10'; Pozantı, Belemedik, P. avium, 22.V.2019, 3\%क, $20^{\prime} 0^{*}$; Pozantı, Karakışlakçı, Fragaria $x$ ananassa, 17.VII.2019, 6\%\%, 50'0'; Pozantı, Karakışlakçı, $P$. armeniaca, 17.VII.2019, 2\%ᄋ, 30"0'; Pozantı, Karakışlakçı,

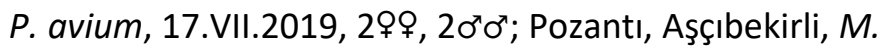
domestica, 17.VII.2019, 60'0'; Pozantı, Belemedik, P. persica, 07.VIII.2019, 3\%०, 30"0'; Pozantı, Karakışlakçı, $M$. domestica, 05.XI.2019, 62\%o, 110 $0^{\top}$; Pozantı, Aş̧̧ıbekirli, P. avium, 05.XII.2019, 119o, 30 $0^{\top} 0^{\top}$; Hatay, Antakya, Merkez, P. persica, 22.VII.2018, 19; Defne, F. carica, 22.VII.2018, 3\%क; Defne, R. fruticosus, 22.VII.2018, 2qo; Kahramanmaras, Andırın, Çiğsar, P. avium, 19.VII.2018, 3\%ᄋ, 10;; Mersin, Çayıryurt, P. persica var. nucipersica, 13.VII.2018, 2\%ᄋ, 10'; Çayıryurt, P. persica, 13.VII.2018, 29\%; Çayıryurt, V. vinifera, 13.VII.2018, 19; Değnek, M. alba, 13.VII.2018, 10'; Değnek, P. cerasus, 13.VII.2018, 19, 10'; Kızılbağ, P. persica, 13.VII.2018, 3우, 200
Huzurkent, V. vinifera, 13.VII.2018, 2\%क; Kepirli, P. domestica, 13.VII.2018, 4\%\%; Osmaniye, Merkez, $R$. fruticosus, 01.VIII.2018, 3\%क; Merkez, F. carica, 01.VIII.2018, 1 ? .

Host: Actinidia chinensis Planch. (Actinidiaceae), A. deliciosa A. Chev., Berberis aquifolium Pursh. (Berberidaceae), C. sinensis, Cornus sp., Cotoneaster lacteus W.W. Sm. (Rosaceae), D. kaki, Elaeagnus umbellate Thunb.(Elaeagnaceae), E. japonica, F. carica, Fragaria $x$ ananassa, Lonicera caerulea L. (Caprifoliaceae), Lindera benzoin L. (Lauraceae), Malpighia emarginata DC. (Malpighiaceae), $M$. domestica, Morus sp., $P$. armeniaca, $P$. avium, $P$. domestica, P. laurocerasus L. (Rosaceae), P. lusitanica L. (Rosaceae), P. persica, Pyrus pyrifolia (Burm.) Nak. (Rosaceae), Rubus spp., R. spectabilis Pursh (Rosaceae), Sambucus nigra L. (Adoxaceae), Solanum dulcamara L. (Solanaceae), S. lycopersicum L. (Solanaceae), S. villosum Mill. (Solanaceae), Vaccinium corymbosum L. (Ericaceae), Vaccinium sp., V. vinifera. (EPPO, 2020a).

Distribution: America, Africa, Asia, Azerbaijan, Europe, Georgia, Oceania (Parshad and Paika, 1964; Hauser, 2011; Paula et al., 2014; Oboňa et al., 2019; Ouantar et al., 2020; EPPO, 2020b).

Distribution in Turkey. Adana, Erzurum, Mersin, Uşak (Orhan et al., 2016; Catal et al., 2018; Zengin, 2020).

Drosophila suzukii, known as the spotted wing drosophila, which presently deserves the cosmopolitan status. The European and Mediterranean Plant Protection Organization (EPPO) has listed D. suzukii on its A2 List. Likely originate of East Asia, this pest has spread to western regions: it was first detected in Hawaii in the early 1980s and following years in Europe and North America (Asplen et al., 2015). Unlike other Drosophila species, the fact that $D$. suzukii can infest the healthy fruits, which have not been damaged by any factor, causes significant economic losses in agriculture. In Turkey, this species was first detected on strawberry crops from eastern Turkey in 2014 (Orhan et al., 2016). In the present study, $D$. suzukii individuals were found at higher altitudes until December on commercial plantations. Zengin (2020) reported that average temperature of October and November with highest number of catch was 5 to $10{ }^{\circ} \mathrm{C}$. The cherry trees, on which the traps were hung, were harvested in June, whereas the apple were harvested in October. Drosophila suzukii cannot live all life stages on the fermenting materials as in other drosophilid and the female individuals need intact fruits for laying their eggs (Cini et al., 2012). For this reason, it is thought that $D$. suzukii having difficulties in finding food tended towards 
the traps containing apple vinegar until December.

\section{Drosophila phalerata Meigen, 1830}

Material examined: Adana, Pozantı, Alpu, P. avium, 22.VI.2017, 19; Saimbeyli, Kalesekisi, P. avium, 24.VII.2018, 2\%ᄋ, 10'; Mersin, Çayıryurt, P. persica, 13.VII.2018, 10'; Huzurkent, V. vinifera, 13.VII.2018, 19; Kızılbağ, P. persica, 13.VII.2018, 2\%ᄋ, 10".

Host: Ageratina adenophora (Spreng.) King \& H.Rob. (Asteraceae), Castanea sativa Mill. (Fagaceae), Clethra arborea Aiton (Clethraceae), Eucalyptus globulus Labill. (Myrtaceae), Juglans regia L. (Juglandaceae), Laurus novocanariensis Rivas Mart., Lousâ, Fern.Prieto, E.Díaz, J.C.Costa \& C.Aguiar (Lauraceae), M. domestica, Musa sp., mushrooms, Ocotea foetens (Aiton) Baill. (Lauraceae), Persea indica (L.) Spreng. (Lauraceae), Pinus pinaster Aiton (Pinaceae), P. armeniaca, P. avium, V. vinifera (Watabe et al., 1993; Rego et al., 2014; Zengin, 2020).

Distribution: Africa, Azores, Caucasus, China, Europe, Iran, Lebanon, Morocco, Russia: East Siberia, West Siberia, Tunisia (Burla, 1951; Watabe et al., 1993; Ivannikov, 1995; Ivannikov et al., 1998).

Distribution in Turkey. Adana, Bursa, Mersin, Samsun, Trabzon, Uşak, Zonguldak (Koçak and Kemal, 2013; Catal et al., 2018; Zengin, 2020).

\section{Genus: Zaprionus Coquillett, 1901}

\section{Zaprionus indianus Gupta, 1970}

Material examined: Adana, Merkez, D. kaki, 10.IX.2017,

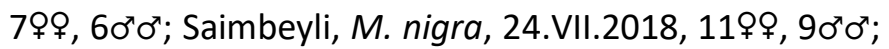
Pozantı, Hamidiye, P. avium, 05.IX.2018, 2\%o, 10'; Pozantı, Belemedik, P. avium, 12.IX.2018, 6\%९, 20"0"; Pozantı, Alpu, P. avium, 23.IX.2018, 5\%ᄋ, 40"o'; Pozantı, Belemedik, P. persica, 17.X.2018, 3\%ᄋ, 70 $0^{\top}$; Pozant, Aşçıbekirli, M. domestica, 31.X.2018, 2\%o; Pozantı, Karakışlakçı, M. domestica, 31.X.2018, 2\%\%; Pozantı, Karakışlakçı, Fragaria x ananassa, 14.XI.2018, 3ㅇ, 10"; Hatay, Defne, R. fruticosus, 22.VII.2018, 16\%?, 80"0'; Defne, F. carica, 22.VII.2018, 21\%ᄋ, 220 $0^{\top}$; Antakya, Merkez, F. carica, 27.VII.2018, 18\%ᄋ, 150 $0^{\pi}$; Mersin, Değnek, P. avium, 13.VII.2018, 4\%9, 30'ơ; Kızılbağ, $P$. persica, 13.VII.2018, 7\%ᄋ, 50'0'; Osmanive, Merkez, $R$. fruticosus, 01.VIII.2018, 25\%9, 210 $0^{\pi}$; Merkez, $P$. domestica, 01.VIII.2018, 199\%, $210^{7} 0^{7}$.

Host: Carica papaya L. (Caricaceae), Diospyros sp., F. carica, $M$. domestica, Mangifera indica L. (Anacardiaceae), Opuntia cordobensis Speg (Cactaceae), $O$. ficus indica Miller (Cactaceae), O. quimilo K. Schum (Cactaceae), P. avium, P. persica, Psidium guajava L.
(Myrtaceae), P. communis, Rubus idaeus L. (Rosaceae), Rubus sp., V. vinifera (Lavagnino et al., 2008; Joshi et al., 2014).

Distribution: Africa, Argentina, Brazil, Central and North America, France, India, Saudi Arabia, South America, Spain, Uruguay (Vilela, 1999; De Toni et al., 2001; van der Linde et al., 2006; Carles-Tolrá, 2009; Kremmer et al., 2017).

Distribution in Turkey. Adana, Hatay, Mersin, Osmaniye (Catal et al., 2019).

Another invasive species, $Z$. indianus, detected in the present study and taken into the alert list by the European and Mediterranean Plant Protection Organization (EPPO) in 2016 (Balmes and Mouttet, 2019) is Zaprionus tuberculatus Malloch and Z. indianus (EPPO, 2016). Both species, in 2020, the Working Party on Phytosanitary Regulations agreed that it could be deleted, considering that sufficient alert has been given (EPPO, 2020c, d).

One of the most successful colonizing species of the genus Zaprionus is $Z$. indianus (Chassagnard and Tsacas, 1993), likely due to its broad niche features: it uses various food resources and shows tolerance to environmental conditions. The first record of $Z$. indianus in Turkey was in the Eastern Mediterranean region (Catal et al., 2019). In our study, we detected $Z$. indianus in apple, cherry, peach, blackberry, fig and plum orchards. In another study, it was reported that $Z$. indianus lays eggs on developing fruits such as figs, peaches and oranges (van der Linde et al., 2006).

\section{Zaprionus tuberculatus Malloch, 1932}

Material examined: Adana, Balcalı, M. alba, 17.VI.2016,

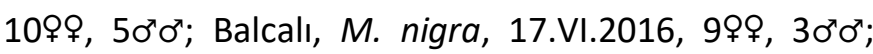
Balcalı, P. avium, 04.V.2018, 3\%९, 10'; Pozantı, Hamidiye, P. avium, 05.IX.2018, 3\%ᄋ, 10; Balcalı, E. japonica, 20.V.2018, 5\%९, 20"0"; Merkez, Fragaria $x$ ananassa, 24.V.2018, 3\%?; Saimbeyli, Merkez, P. avium, 29.VI.2018, 5\%ᄋ, 40 $0^{\pi}$; Saimbeyli, Obruk, P. avium, 29.VI.2018, 69\%; Tufanbeyli, P. avium, 29.VI.2018, 5\%ᄋ, 10'; Saimbeyli, Merkez, R. fruticosus, 24.VII.2018, 39\%, $20^{\top} 0^{\pi}$; Saimbeyli, Kalesekisi, M. alba, 24.VII.2018, 29\%, 20"0'; Saimbeyli, Kalesekisi, P. avium, 24.VII.2018, 5ㅇ, 30"0'; Pozantı, Belemedik, P. avium, 12.IX.2018, 20\%ᄋ, $30^{\top} 0^{\prime}$; Pozantı, Alpu, P. avium, 23.IX.2018, 49\%, 50"0'; Pozantı, Belemedik, P. persica, 24.X.2018, 59ᄋ, 10;; Pozantı, Aşçıbekirli, M. domestica, 31.X.2018, 39\%; Pozantı, Karakışlakçı, M. domestica, 31.X.2018, 20"0"; Pozantı, Karakışlakçı, Fragaria x ananassa, 14.XI.2018, 1\%, 10'; Balcalı, P. domestica, 02.VII.2019, 11\%o, 60'0'; 
Pozantı, Karakışlakçı, P. avium, 10.X.2019, 19; Hatay

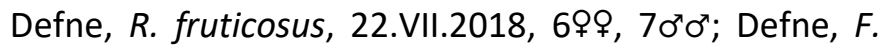
carica, 22.VII.2018, 10\%९, 150"o'; Defne, P. domestica, 22.VII.2018, 5\%ᄋ, 40"0'; Kahramanmaras, Geben, $P$. cerasus, 19.VII.2018, 3\%९, 10'; Andırın, Çiğsar, P. avium, 19.VII.2018, 4\%̊; Andırın, Çokak, P. avium, 19.VII.2018, 4\%९,50 $0^{\top}$; Mersin, Merkez, P. persica var. nucipersica, 15.VI.2018, 4\%ᄋ, $20^{\top} 0^{\top}$; Merkez, P. domestica, 21.VI.2018,

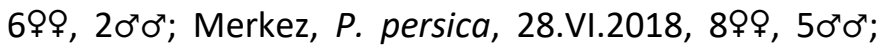

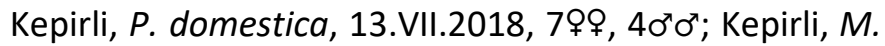
alba, 13.VII.2018, 5\%ᄋ; Değnek, P. avium, 13.VII.2018,

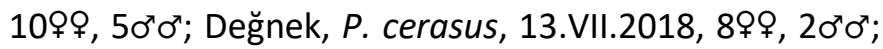
Huzurkent, P. domestica, 13.VII.2018, 7\%, 120 $0^{\top} 0^{\pi}$; Huzurkent, V. vinifera, 13.VII.2018, 6\%९, $50^{\top} 0^{\top}$; Çayıryurt, V. vinifera, 13.VII.2018, 4\%ᄋ; Çayıryurt, P. communis, 13.VII.2018, 1우, 30'0 $0^{\top}$; Çayıryurt, P. persica, 13.VII.2018,

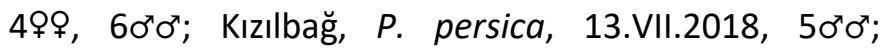
Değirmençay, P. persica, 13.VII.2018, 19, 40'o'; Gözne, M. domestica, 13.VII.2018, 3\%?; Merkez, R. fruticosus, 16.VII.2018, 5\%?; Merkez, P. domestica, 16.VII.2018, 3ㅇ, 10'; Osmaniye, Merkez, V. vinifera, 01.VIII.2018,

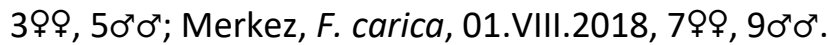

Host: Artocarpus sp. (Moraceae), citrus garden, Dacryodes sp. (Burseraceae), Detarium senegalense J.F.Gmel. (Fabaceae), Ficus lutea Vahl (Moraceae), F. mucuso Welw. ex Ficalho, F. natalensis Hochst., F. saussureana DC., F. sur Forssk., Gambeya taiensis Aubrév. \& Pellegr. (Sapotaceae), Guarea cedrata (A. Chev.) Pellegrin (Meliaceae), Hirtella sp. (Chrysobalanaceae), Opuntia fruit, Parinari excelsa Sabine (Chrysobalanaceae), Santiria trimera (Oliv.) Aubrév. (Burseraceae), Spondias mombin L. (Anacardiaceae), Tieghemella heckelii (A.Chev) Pierre ex Dubard (Sapotaceae), Uncaria sp. (Rubiaceae) (Ebejer, 2001; EPPO, 2020c).

Distribution: Africa: Cameroon, Cape Verde, Central African Republic, Chad, Congo, Congo (Democratic republic), Cote d'Ivoire, Egypt, Gabon, Kenya, Madagascar, Malawi, Mauritius, Mayotte, Mozambique, Niger, Nigeria, Réunion, Saint Helena, Seychelles, South Africa, Tanzania, Uganda, Zambia, Zimbabwe, EPPO region: Azerbaijan, Cyprus, Egypt, Georgia, Greece (Crete), Israel, Italy, Malta, Spain (Islas Canarias only), Romania (Oboňa et al., 2019; EPPO, 2020c).

Distribution in Turkey. Adana, Uşak (Patlar et al., 2012; Zengin, 2020).
Zaprionus tuberculatus, which is native to the Afrotropical region, has been detected in Turkey for the first time in Adana province in 2011 (Patlar et al., 2012). Patlar et al. (2012) and Zengin (2020) reported regarding the detection of $Z$. tuberculatus in August. However, since no sampling has been performed in the months before and after August, the status of $Z$. tuberculatus in these months was unknown. This species has been seen in fruit and traps from May to November in our study. Constantina et al. (2015) detected this species in Romania for the first time in 2014 and they reported that Z. tuberculatus has been found in the traps from late September to late October. EPPO (2020c) reported that it is difficult to assess the pest potential of $Z$. tuberculatus against healthy fruit varieties because little is known about its biology and ecology. However, it has been said that, along with other primary pests (such as D. suzukii observed in Romania), these pests can contribute to enhancing the negative effects on fruit crops.

\section{Subfamily: Steganinae \\ Genus: Leucophenga Mik, 1886 \\ Leucophenga maculata Dufour, 1839}

Material examined: Adana, Pozantı, Hamidiye, P. avium, 09.V.2019, 19, 20'0'; Pozantı, Belemedik, P. avium, 17.V.2019, 3ㅇ, 10'; Mersin, P. cerasus, 16.VII.2018, 1\%, $10^{\circ}$.

Host: M. domestica, Musa sp., mushrooms, $P$. armeniaca, P. avium, V. vinifera, (Watabe et al., 1993; Zengin, 2020).

Distribution: Azerbaijan, China, Europe, Georgia, Japan, Korea, Sri Lanka, Turkmenistan (Okada, 1956; Watabe et al., 1993; Gornostaev, 1997; Oboňa et al., 2019).

Distribution in Turkey. Mersin, Samsun, Şırnak, Uşak (Koçak and Kemal, 2013; Zengin, 2020).

\section{Genus: Gitona Meigen, 1830}

\section{Gitona distigma Meigen, 1830}

Material examined: Adana, Pozant, Belemedik, $P$. avium, 15.V.2019, 19; Pozantı, Alpu, P. avium, 11.VII.2019, 2 \%o.

Host: P. communis, V. vinifera (Zengin and Karaca, 2019). Distribution: Caucasus, China, Europe, Kazakhstan, Mongolia, Russia: Siberia (Duda, 1934; Okada, 1973; Watabe et al., 1993; Ivannikov et al., 1998).

Distribution in Turkey. Hakkari, Şırnak, Uşak (Koçak and Kemal, 2013; Zengin and Karaca, 2019). 


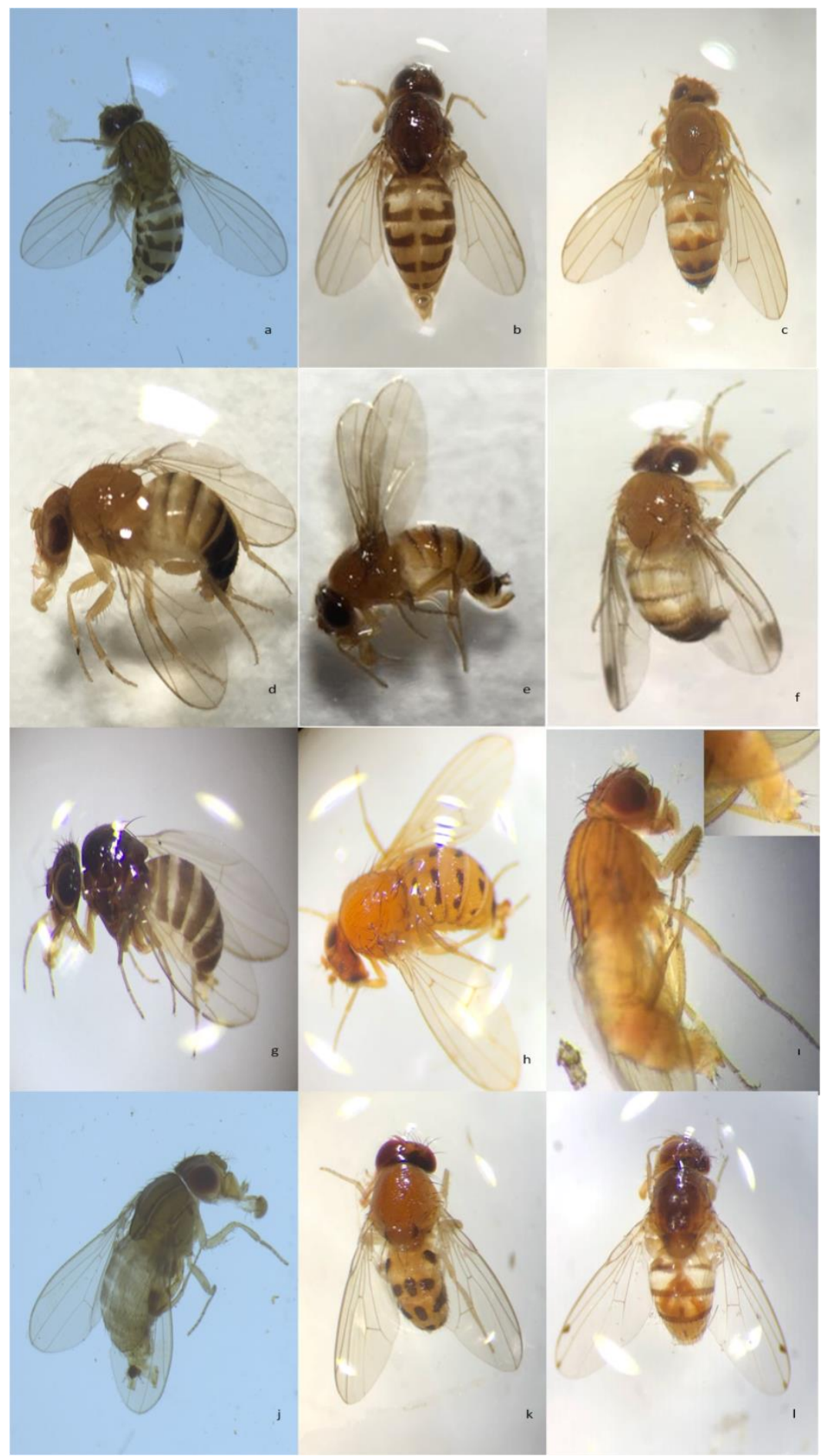

Figure 2. General view. Drosophila busckii (a), Drosophila hydei (b), Drosophila immigrans (c), Drosophila melanogaster (d), Drosophila suzukii female (e), and male (f), Drosophila subobscura (g), Drosophila phalerata $(\mathrm{h})$, Zaprionus indianus (I), Zaprionus tuberculatus (j), Leucophenga maculata (k), Gitona distigma (I)

In conclusion, most of the drosophilids obtained in this study are thought to be widely dispersed natural populations in Turkey biomes, and two Drosophilidae species recently recorded Turkey: $D$. suzukii, first noted in 2014 (Orhan et al., 2016) and Zaprionus indianus, in 2019 (Catal et al., 2019). Although Zaprionus is a different genus, the genus Drosophila is paraphyletic with Zaprionus (van der Linde et al., 2010). These species are particularly interest because, in contrast to most drosophilids, they are agricultural pests.

These results emphasizes the importance of scientific collections as a source of data on regional biodiversity. Most of the records are material collected with fruit and traps. It is possible that the list conferred here can be 
developed not only by collecting in places that have not been sampled, but also by using other sources, such as decomposing material, fungi, flowers and other attractants.

\section{ÖZET}

Amaç: Bu çalışmanın amacı, Türkiye'nin Doğu Akdeniz Bölgesi'ndeki meyve bahçelerinde Drosophilidae familyası türlerinin varlığını belirlemektir.

Yöntem ve Bulgular: Sörveyler, 2016-2019 yılları arasında Türkiye'nin Doğu Akdeniz Bölgesi'nde (Adana, Hatay, Kahramanmaraş, Mersin, Osmaniye) çeşitli meyve bahçelerinde yürütülmüştür. Drosophilid türleri farklı meyvelerden toplanmış ve teşhis edilmiştir. Çalışma sonucunda, 4 cinse bağlı 11 tür tanılanmış olup, bunların çoğu, oldukça istilacı ve özellikle meyve kültürlerini tehdit eden türlerdir. Sonuç olarak, Drosophila immigrans (Sturtevant), D. melanogaster (Meig.), D. subobscura (Collin), D. suzukii (Matsumura), Zaprionus indianus (Gupta) ve Z. tuberculatus (Gupta) türleri Doğu Akdeniz Bölgesi'nde yaygın olarak bulunmuştur.

Genel Yorum: Bu çalışmada elde edilen çoğu Drosophilid türünün, Türkiye biyomlarında yaygın olarak dağılmış doğal popülasyonlar olduğu düşünülmektedir ve bunların arasında Türkiye'de yakın zamanda kaydedilen iki Drosophila türü bulunmaktadır.

Çalışmanın Önemi ve Etkisi: Bu sonuçlarla bilimsel koleksiyonlar ile bölgesel biyoçeşitlilik çalışmalarının önemi vurgulanmaktadır.

Anahtar Kelimeler: Drosophilidae, Drosophila, Zaprionus, Türkiye.

\section{ACKNOWLEDGEMENTS}

We would like thanks to Amir Yassin (CNRS researcher, Institut de Systématique, Evolution, Biodiversité / France) for the diagnosis of drosophilid species. This study was supported by Çukurova University as scientific research project numbered FBA-2016-6528. Part of this study is presented as poster in 4th International Agriculture Congress (5-8 July 2018, Nevşehir, Turkey) and published as an abstract in meeting book.

\section{CONFLICT OF INTEREST}

The authors declare no conflict of interest for this study.

\section{AUTHOR'S CONTRIBUTIONS}

The contribution of the authors is equal.

\section{REFERENCES}

Anonymous (2020) Retrieved December 25, 2020 from https://www.google.com.tr/maps/@37.2725-

$721,35.2883658,268535 \mathrm{~m} /$ data $=! 3 \mathrm{~m} 1 ! 1 \mathrm{e} 3$

Asplen MK, Anfora G, Biondi A, Choi DS, Chu D, Daane KM, Gibert P, Gutierrez AP, Hoelmer KA, Hutchison WD, Isaacs R, Jiang ZL, Kárpáti Z, Kimura MT, Pascual $M$, Philips CR, Plantamp C, Ponti L, Vétek $G$, Vogt $H$, Walton VM, Yu Y, Zappalà L, Desneux N (2015) Invasion biology of spotted wing Drosophila (Drosophila suzukii): a global perspective and future priorities. J. Pest Sci. 88: 469-494.

Bächli G (2020) TaxoDros: The database on taxonomy of Drosophilidae. Retrieved January 08, 2021 from Eletronic Database accessible at http://www.taxodros.uzh.ch/

Bächli G, Burla H (1985) Insecta Helvetica Fauna. Vol. 7: Diptera: Drosophilidae, /16 pp. Schweiz. Ent. Ges. Zürich.

Bächli G, Rocha Pité MT (1984) Family Drosophilidae, pp 186-220. In: Catalogue of Palaearctic Diptera. Vol.10. Clusiidae-Chloropidae. Akadémiai Kiadó, Budapest.

Bächli G, Vilela CR, Haring E (2002) Four new species of west Palaearctic Drosophilidae (Diptera). Mitteilungen der Schweizerischen Entomologischen Gesellschaft 75(3/4): 299-333.

Balmes V, Mouttet R (2019) The drosophilid risk on imports. EPPO Bulletin 49: 122-126.

Brake I, Bachli G (2008) Drosophilidae (Diptera). World Catalogue of Insects Vol. 9, 412 pp.

Burla H (1951) Systematik, Verbreitung und Oekologie der Drosophila-Arten der Schweiz. Rev. Suisse Zool. 58: 23-175.

Capy P, Gibert P (2004) Drosophila melanogaster, Drosophila simulans: so similar yet so different. In Drosophila melanogaster, Drosophila simulans: So Similar, So Different. Springer, Dordrecht. pp. 5-16.

Carles-Tolrá M (2009) Zaprionus indianus Gupta: género y especie nuevos para la Península Ibérica (Diptera: Drosophilidae). Boletín de la Sociedad Entomológica Aragonesa 45: 316.

Çatal BO, Kece AFC, Ulusoy MR (2018) Drosophilidae Fauna in Eastern Mediterranean Region with A New Record from Turkey. 4. International Agriculture Congress, 05-08 July, 2018, Nevşehir-Turkey. p. 201.

Çatal BO, Kece AFC, Ulusoy MR (2019) New invasive species in Turkey: Zaprionus indianus (Gupta) (Diptera: Drosophilidae). Kahramanmaraş Sütçü Imam Üniversitesi Tarım ve Doğa Dergisi 22: 110-113. 
Chassagnard MT, Tsacas L (1993) The Subgenus Zaprionus-s. str. definition of species groups and revision of the Vittiger subgroup (Diptera, Drosophilidae). Annales de la Societe Entomologique de France 29: 173-194.

Cini A, loriatti C, Anfora G (2012) A review of the invasion of Drosophila suzukii in Europe and a draft research agenda for integrated pest management. Bulletin of Insectology 65(1): 149-160.

Constantina C, Teodoru A, Chiriloaie A (2015) The first detection of fruit fly Zaprionus tuberculatus Malloch (Diptera: Drosophilidae) in the eastern part of Europe (Romania). University of Cracovia 20: 377-382.

David JR, Tsacas L (1981) Cosmopolitan, subcosmopolitan and widespread species: different strategies within the Drosophilidae family (Diptera). C. R. Séances Soc. Biogeogr. 57: 11-26.

De Toni DC, Hofmann PR, Valente VLS (2001) First record of Zaprionus indianus (Diptera, Drosophilidae) in the state of Santa Catarina, Brazil. Biotemas 14(1): 71-85.

Dobzhansky T (1965) 'Wild', and 'Domestic', Species of Drosophila', in Baker, H.G., and Stebbins, G.L. (eds.), The Genetics of Colonizing Species, Academic Press, New York, pp. 533-546.

Duda O (1934) Drosophilidae. In: Lindner, E. (ed.). Die Fliegen der palaearktischen Region. Part 1. Stuttgart: 1-64.

Ebejer MJ (2001) A contribution to the knowledge of the Ephydroidea (Diptera: Camillidae, Campichoetidae and Drosophilidae) of Malta. The Central Mediterranean Naturalist 3: 85-88.

EPPO (2016) Retrieved January 12, 2021 from https://gd.eppo.int/reporting/article-5952

EPPO (2020a) Retrieved December 25, 2020 from https://gd.eppo.int/taxon/DROSSU/hosts

EPPO (2020b) Retrieved December 25, 2020 from https://gd.eppo.int/taxon/DROSSU/distribution

EPPO (2020c) Mini datasheet on Zaprionus tuberculatus (Diptera: Drosophilidae). Retrieved December 25, 2020 from https://gd.eppo.int/taxon/ZAPRTU/documents

EPPO (2020d) Mini datasheet on Zaprionus indianus (Diptera: Drosophilidae). Retrieved December 25, 2020 from https://gd.eppo.int/taxon/ZAPRIN/documents

Gençer N, Coşkuncu K, Kumral N (2005) Bursa ilinde incir bahçelerinde görülen zararlı ve yararlı türlerin saptanması. Anadolu Tarım Bilimleri Dergisi 20(2): 2430.
Gibert P, Hill M, Pascual M, Plantamp C, Terblanche JS, Yassin A, Sgrò CM (2016) Drosophila as models to understand the adaptive process during invasion. Biol. Invasions 18: 1089-1103.

Gleason JM, Roy PR, Everman ER, Gleason TC, Morgan TJ (2019) Phenology of Drosophila species across a temperate growing season and implications for behavior. Plos ONE 14(5): 1-21.

Gornostaev NG (1997) Addition to the fauna of drosophilid flies (Diptera, Drosophilidae) of Russia. Russian Entomological Journal 6(1-2): 113-118.

Goto SG, Yoshıda T, Beppu K, Kimura MT (1999) Evolution of overwintering strategies in Eurasian species of the Drosophila obscura species group. Biological Journal of the Linnean Society 68(3): 429441.

Guo J (2007) Bionomics of fruit flies Drosophila melanogaster damage cherry in Tianshui. Chinese Bulletin of Entomology 44: 743-745.

Hauser M (2011) A historic account of the invasion of Drosophila suzukii (Matsumura) (Diptera: Drosophilidae) in the continental United States, with remarks on their identification. Pest Management Science 67(11): 1352-1357.

Hochmüller CJ, Lopes-da-Silva M, Valente VL, Schmitz HJ (2010) The drosophilid fauna (Diptera, Drosophilidae) of the transition between the Pampa and Atlantic Forest Biomes in the state of Rio Grande do Sul, southern Brazil: first records. Papéis Avulsos de Zoologia 50(19): 286-295.

Hoffmann AA, Sorensen JG, Loeschcke V (2003) Adaptation of Drosophila to temperature extremes: bringing together quantitative andmolecular approaches. Journal of Thermal Biology 28: 175-216.

Ivannikov AV (1995) Drosophila species from forest mushrooms in Novosibirsk vicinity. Drosophila Information Service 76: 138.

Ivannikov AV, Katokhin AV, Sidorenko VS (1998) List of Drosophilidae (Diptera) of West Siberia. Far Eastern Entomologist 57: 12-16.

Ivannikov AV, Zakharov IK (1994) Synantropic species of Drosophila (Diptera: Drosophilidae) in some regions of Eurasia. Drosophila Information Service 75: 108109.

Joshi NK, Biddinger DJ, Demchak K, Deppen A (2014) First report of Zaprionus indianus (Diptera: Drosophilidae) in commercial fruits and vegetables in Pennsylvania. Journal of Insect Science 14: 1-4.

Koçak AÖ, Kemal M (2013) Diptera of Turkey. Priamus Supplement 28: 1-411. 
Kremmer L, David J, Borowiec N, Thaon M, Ris N, Poirié M, Gatti JL (2017) The African fig fly Zaprionus indianus: a new invasive pest in France. Bulletin of Insectology 70(1): 57-62.

Langille $A B$, Arteca EM, Ryan GD, Emiljanowicz LM, Newman JA (2016) North American invasion of Spotted-Wing Drosophila (Drosophila suzukii): A mechanistic model of population dynamics. Ecological Modelling 336: 70-81.

Lavagnino NJ, Carreira VP, Mensch J, Hasson E, Fanara JJ (2008) Geographic distribution and hosts of Zaprionus indianus (Diptera: Drosophilidae) in NorthEastern Argentina. Revista de la Sociedad Entomologica Argentina 67: 189-192.

Lewontin RC (1965) Selection for colonizing ability. In: Baker, H.G., Stebbins, G.L. (Eds.), The Genetics of Colonizing Species. Academic Press, London, pp. 7791.

Li D, Chen X, Yuan J, Hu J (2011) Biological character observation and insecticide screening of Drosophila melanogaster on cherry. Guizhou Agricultural Science 39: 92-94.

Máca J (1987) Amiota (Phortica) goetzi sp. n. (Diptera, Drosophilidae) with faunistic notes to Drosophilidae, Odinidae and Periscelididae from southeastern Europe and Turkey. Acta Entomologica Musei Nationalis Pragae 42: 311-320.

Markow TA, O'grady P (2008) Reproductive ecology of Drosophila. Functional Ecology 22(5): 747-759.

Nartshuk EP (2014) Fruit flies (Diptera: Drosophilidae) of the Russian Arctic. Zoosystematica Rossica 23(2): 256-263.

Oboňa J, Dvořák L, Haenni JP, Hrivniak L', Japoshvili B, Ježek J, Manko P (2019) New and interesting records of Diptera from Azerbaijan and Georgia. Zoosystematica rossica 28(2): 277-295.

O'Grady PM, DeSalle R (2018) Phylogeny of the genus Drosophila. Genetics 209(1): 1-25.

Okada T (1954) Comparative morphology of the drosophilid flies. I. Phallic organs of the melanogaster group, Kontyu, 22: 36-46.

Okada T (1956) Systematic study of Drosophilidae and allied families of Japan. Gihodo, Tokyo, 283 pp.

Okada T (1973) Drosophilidae and Diastatidae from Mongolia (Diptera). Ann Hist-nat Mus Nat Hung. 65: 271-279.

Orhan A, Aslantaş R, Önder BŞ, Tozlu G (2016) First record of the invasive vinegar fly Drosophila suzukii (Matsumura)(Diptera: Drosophilidae) from eastern Turkey. Turkish Journal of Zoology 40(2): 290-293.
Ouantar M, Anfora G, Bouharoud R, Chebli B (2020) First report of Drosophila suzukii (Diptera: Drosophiladae) in North Africa. Moroccan Journal of Agricultural Sciences 1(5): 277-279.

Parshad R, Paika IJ (1964) Drosophilid survey of India. II. Taxonomy and cytology of the subgenus Sophophora (Drosophila). Research Bulletin of Punjab University 15: 225-252.

Parsons PA (1983) Ecobehavioral genetics: habitats and colonists. Annu. Rev. Ecol. Syst. 14: 35-55.

Patlar B, Koc B, Yilmaz M, Ozsov ED (2012) First records of Zaprionus tuberculatus (Diptera: Drosophilidae) from the Mediterranean Region, Turkey. Drosophila Information Service 95: 94-96.

Paula MA, Lopes PHS, Tidon R (2014) First record of Drosophila suzukii in the Brazilian Savanna. Drosophila Information Service 97: 113-115.

Rego C, Boieiro M, Gonçalves Y, Menezes D, AguinPombo D, Capela RA (2014) The drosophilids (Diptera: Drosophilidae) from a Laurisilva patch in Madeira with two new records for this island. Bocagiana 238: 1-8.

Sene FM, Val FC, Vilela CR, Pereira MAQR (1980) Preliminary data on the geographical distribution of Drosophila species within morpho-climatic domains of Brazil. Papéis Avulsos de Zoologia 33: 315-326.

Stephens AR, Asplen MK, Hutchison WD, Venette RC (2015) Cold hardiness of winter-acclimated Drosophila suzukii (Diptera: Drosophilidae) adults. Environmental Entomology 44(6): 1619-1626.

Sturtevant AH (1942) The classification of the genus Drosophila, with descriptions of nine species. Univ. Texas Publ. 4213: 5-51.

Toda MJ (1986) Drosophilidae (Diptera) in Burma. II: The Drosophila immigrans species group of the subgenus Drosophila. Kontyū (Tokyo), 54(4): 634-653.

Van der Linde K, Houle D, Spicer GS, Steppan SJ (2010) A supermatrix-based molecular phylogeny of the family Drosophilidae. Genetics Research 92(1): 25-38.

Van der Linde K, Steck GJ, Hibbard K, Birdsley JS, Alonso LM, Houle D (2006) First records of Zaprionus indianus (Diptera: Drosophilidae), a pest species on commercial fruits from Panama and the United States of America. Florida Entomologist 89: 402-404.

Vilela CR, Mori L (1999) The genus Drosophila (Diptera, Drosophilidae) in the Serra do Cipó: further notes. Revista Brasileira de Entomologia 43(3/4): 319-328.

Watabe H, Toda MJ, Li GC, Duan CL, Imitty R, Entomack B, Muhtar A (1993) Drosophilid fauna (Diptera, Drosophilidae) of Chinese Central Asia. Jpn. J. Ent. 61: 525-545. 
Zengín E (2020) Occurrence of invasive species and seasonal dynamics of fruit flies (Diptera: Drosophilidae) species in Uşak province, Turkey. Revista de la Sociedad Entomológica Argentina 79(1): 21-30.
Zengin E, Karaca i (2019) Dynamics of trapped adult populations of Drosophila suzukii Matsumura (Diptera: Drosophilidae) and its parasitoids in Uşak Province, Turkey. Egypt J. Biol. Pest Control 29: 43. 